\title{
Polarization fraction measurement in same-sign $W W$ scattering using deep learning
}

\author{
Junho Lee, ${ }^{1}$ Nicolas Chanon, ${ }^{2}$ Andrew Levin, ${ }^{1}$ Jing Li,${ }^{1}$ Meng Lu, ${ }^{1}$ Qiang Li, ${ }^{1}$ and Yajun Mao ${ }^{1}$ \\ ${ }^{1}$ Department of Physics and State Key Laboratory of Nuclear Physics and Technology, \\ Peking University, Beijing 100871, China \\ ${ }^{2}$ Institut de Physique Nucléaire de Lyon, Université de Lyon, \\ Université Claude Bernard Lyon 1, CNRS-IN2P3, Villeurbanne 69622, France
}

(Received 18 December 2018; published 8 February 2019)

\begin{abstract}
Studying the longitudinally polarized fraction of $W^{ \pm} W^{ \pm}$scattering at the LHC is crucial to examine the unitarization mechanism of the vector boson scattering amplitude through Higgs and possible new physics. We apply here for the first time a deep neural network classification to extract the longitudinal fraction. Based on fast simulation implemented with the Delphes framework, significant improvement from a deep neural network is found to be achievable and robust over all dijet mass region. A conservative estimation shows that a high significance of four standard deviations can be reached with the High-Luminosity LHC designed luminosity of $3000 \mathrm{fb}^{-1}$.
\end{abstract}

DOI: $10.1103 /$ PhysRevD.99.033004

The High-Luminosity LHC (HL-LHC) will measure for the first time many novel processes predicted by standard model (SM), and study precisely especially those involving pure electroweak interactions such as vector boson scattering (VBS). VBS is sensitive to non-Abelian weak gauge boson interactions, and to the structure of electroweak symmetry breaking. Typical VBS signatures at hadron colliders include, for example, large dijet mass $\left(m_{\mathrm{jj}}\right)$ and large pseudorapidity separation $\left(\Delta \eta_{j j}\right)$.

Among various VBS processes, same charge $W^{ \pm} W^{ \pm}$ production is one of the most promising channels for the above mentioned purpose. The VBS $W^{ \pm} W^{ \pm}$process profits from low background, due to the signature of two same sign charged leptons. Same charge $W^{ \pm} W^{ \pm}$scattering has been observed by CMS and ATLAS with a significance larger than 5 standard deviations, based on data collected at $\sqrt{s}=13 \mathrm{TeV}$, corresponding to an integrated luminosity of approximately $35.9 \mathrm{fb}^{-1}[1,2]$. The dominant backgrounds after the VBS selection arise from WZ production with one lepton misidentified, and nonprompt leptons from hadron decays, which can be further suppressed by requiring $m_{\mathrm{jj}}$ to be above $1 \mathrm{TeV}$.

The next important goal after the discovery of VBS $W^{ \pm} W^{ \pm}$is to measure the fraction of longitudinally polarized (LL) events. The LL component contributes only to a

Published by the American Physical Society under the terms of the Creative Commons Attribution 4.0 International license. Further distribution of this work must maintain attribution to the author(s) and the published article's title, journal citation, and DOI. Funded by SCOAP . level of $5-10 \%$ in $W^{ \pm} W^{ \pm} \rightarrow W^{ \pm} W^{ \pm}$, but it is extremely interesting as a direct probe of the unitarization mechanism [3] of the vector boson scattering amplitude through Higgs and possible new physics [4].

There have been extensive studies on LL fraction measurement, exploiting various kinematic observables. Popular variables include leading lepton transverse momentum $\left(p_{T}^{l_{1}}\right)$, and the azimuthal angle difference between the two leading jets $\left(\Delta \phi_{\mathrm{jj}}\right)$. On top of these, Ref. [5] proposed to use the variable $R_{p_{T}}=\frac{p_{T}^{l_{1}} \cdot p_{T}^{l_{2}}}{p_{T}^{j_{1}} \cdot p_{T}^{j_{2}}}$. Reference [6] examined matrix element method to differentiate different beyond SM model scenarios. More recently, Ref. [7] applied a regression with a deep neural network (DNN) to recover the lepton angular distributions in the $\mathrm{W}$ boson rest frame, and shows that the expected accuracy can be improved by about a factor of two compared to the use of $R_{p_{T}}$.

In the meantime, CMS studied the prospects for a measurement of the LL fraction, based on full simulation samples with the upgraded CMS detector at the $14 \mathrm{TeV}$ HL-LHC $[8,9]$. The expected significance for an integrated luminosity of $3000 \mathrm{fb}^{-1}$ is estimated to be 2.7 standard deviations. The study is based on a fit to $\Delta \phi_{\mathrm{jj}}$ distributions in two $m_{\mathrm{jj}}$ bins.

In this study, we examine the impact of using a DNN on LL fraction measurement. In contrast to what has been done in Ref. [7], we exploit here DNN classification instead of regression, based on the framework of the Keras library [10] with Tensorflow back-end [11]. We perform a fit on the resulting DNN discriminant. 
There have been more and more applications of machine learning techniques in high energy physics, with some first examples in Refs. [12,13]. Detailed studies are provided in this paper based on either low-level or high-level features. A comparison with boosted-decision trees [14] implemented in TMVA [15] are also provided.

Simulation samples are generated with MadGraph5_ aMC@ NLO [16] interfaced with PYTHIA 6 [17] for parton showering and hadronization and DELPHES version 3 [18] for detector simulation with CMS configuration. Similarly as in Ref. [7], we neglect the "pileup" effects due to overlapping interactions in proton proton collision, as they can be mitigated effectively with advanced experimental techniques. The inclusive $W^{ \pm} W^{ \pm}$VBS samples are decomposed into LL, TT (transversely polarized $W^{ \pm} W^{ \pm}$) and TL (transversely and longitudinally polarized $W^{ \pm} W^{ \pm}$) components, with the help of DECAY package provided by MADGRAPH. We require exactly 2 same-sign charged leptons with $p_{T}>20 \mathrm{GeV}$ and $|\eta|<2.4$, and select the two leading jets with $p_{T}>50 \mathrm{GeV}$ and $|\eta|<4.7$ as VBS jet candidates. We further require $\left|\Delta \eta_{\mathrm{jj}}\right|>2.5$, a b jet veto, and performed our studies in several benchmark selections: $m_{\mathrm{jj}}>850,1200,1500,1800$, and $2000 \mathrm{GeV}$. The backgrounds from WZ and nonprompt leptons can indeed be suppressed effectively with higher $m_{\mathrm{jj}}$.

As inputs to the DNN, low-level features include the $p_{T}$, $\eta, \phi$ of the two leptons, $p_{T}, \eta, \phi$ and mass of the two jets, and $\mathrm{x}$ - and $\mathrm{y}$ - components of missing transverse energy $\left(\mathbb{E}_{T}\right)$. We further include high-level features with zeppenfeld variable [19] of the two leptons, $\Delta \phi_{\mathrm{jj}}, \Delta \eta_{\mathrm{jj}}$ and $\Delta \mathrm{R}_{\mathrm{ll}, \mathrm{jj}}$. Four million events have been produced for training, validation and testing. Overtraining has been carefully checked by monitoring loss value dependency on DNN training epoch, in both the training and validation dataset. Early stopping has been applied if there is no improvement in loss value comparing with any latest 20 epoch's loss value. Overtraining can also be precisely checked by comparing output distribution of training and test dataset.

Two differently structured DNN models, a "dense" and a "particle-based" model, have been trained and tested. We selected a 10-layers dense neural network with 150 hidden units on each layer with the "relu" activation function, the "sigmoid" function applied on final nodes, taking "adam" optimizer with a learning rate of 0.001 , and 0.01 as regularization term for L2 regularization. Moreover, a batch size of 50 events, a 50\% drop-out rate on hidden unit, and batch normalization are applied to avoid overtraining. As an alternative, to efficiently model highly correlated variables of each particle, we also tried a particlebased model which involves separate grouping of nodes for the features of each particle and a gradual merging of all nodes into bigger layers. Figure 1 shows a simplified version of the particle-based model. The model actually used contains 2 hidden layers with 20 nodes for each particle. Leptons and jets are merged with 2 layers of

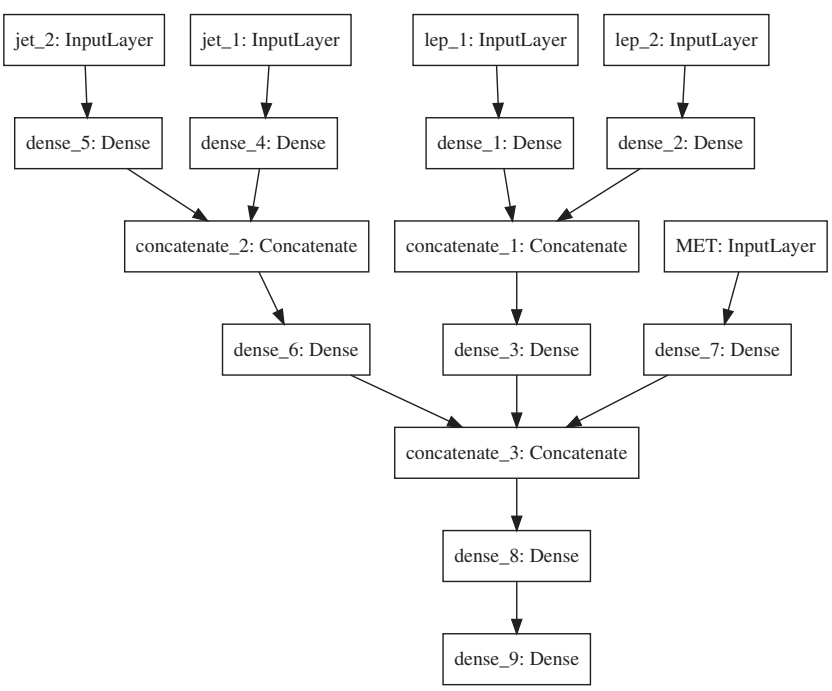

FIG. 1. Simplified structure of the particle-based DNN model. Inputs are features of each particle, gradually merging to the output layers.

40 nodes before they are merged with the $\mathbb{E}_{T}$ features. Finally, 4 layers of 180 nodes are added. Figure 2 shows the receiver operating characteristics (ROC) curve, which has been widely used as a measure of performance. From Fig. 2, improved performance can be found for the particlebased model compared to the dense model. Studies using a DNN model including low and high-level features have been performed, but no significant improvement was found.

Similar studies have been performed using a BDT, with 1000 trees of 5 maximum depth, and "Adaptive Boost" algorithm. Figure 2 shows the performance of each discriminant variable. Calculated area under curve (AUC) is $0.788,0.762,0.776,0.666$, and 0.591 for the

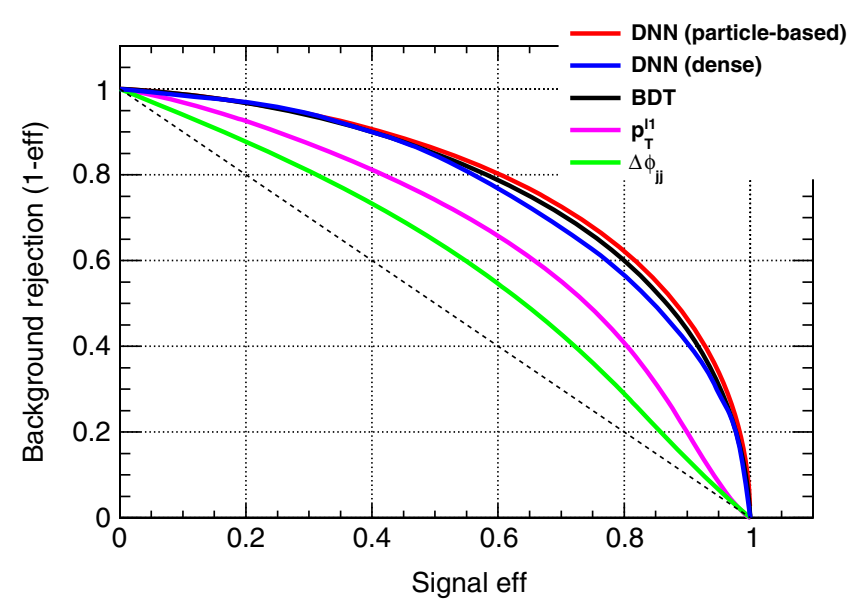

FIG. 2. ROC curves with a selection of $m_{\mathrm{jj}}>1500 \mathrm{GeV}$ applied to VBS $W^{ \pm} W^{ \pm}$. The $\mathrm{X}$-axis showing signal efficiency of LL component, and Y-axis the rejection rate of $\mathrm{TT}+\mathrm{TL}$ components. 


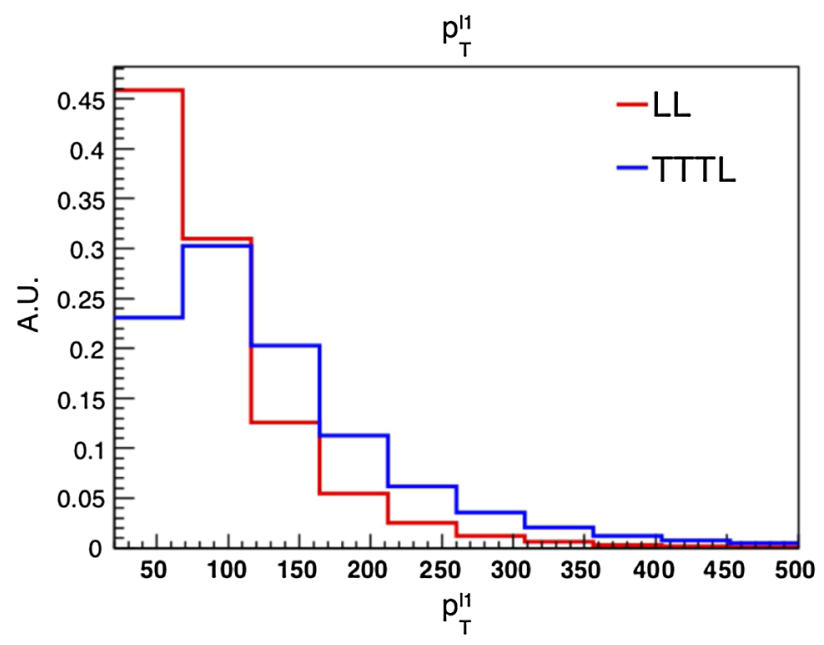

(a)

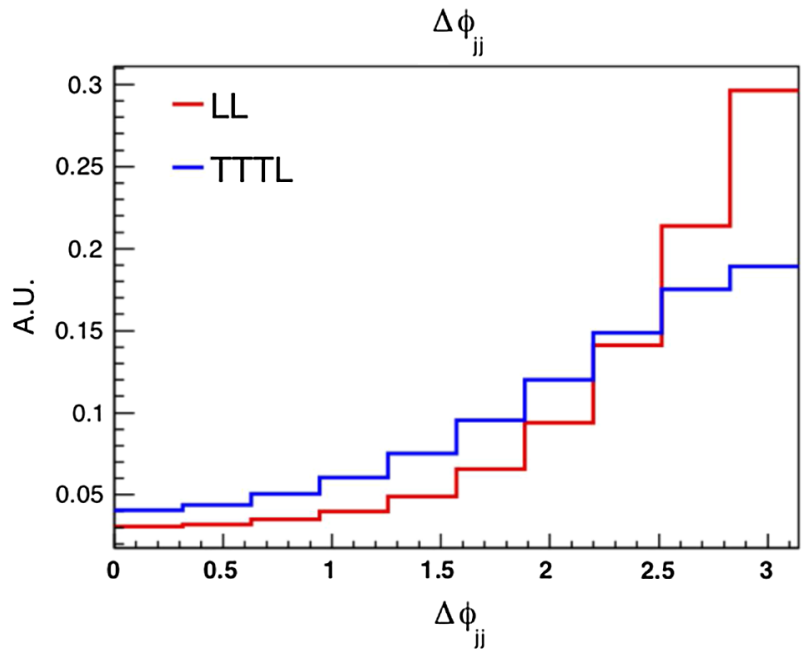

(b)

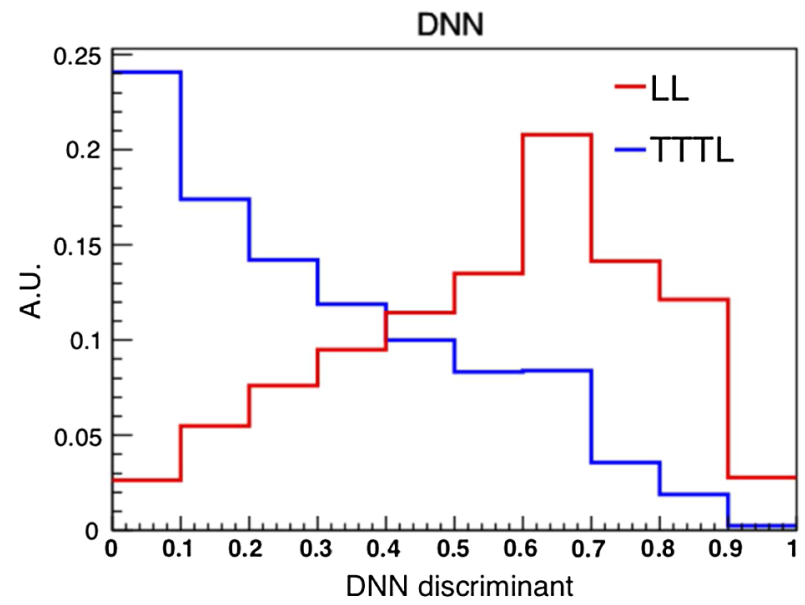

(c)

FIG. 3. Kinematic distributions for $p_{T}^{l_{1}}$ (a), $\Delta \phi_{\mathrm{jj}}$ (b), and DNN discriminant corresponding to the particle-based model (c).
TABLE I. Fit results for LL fraction with varaious $m_{\mathrm{jj}}$ cuts, at $68 \%$ confidence level. True fraction is the LL fraction computed at generator level.

\begin{tabular}{lcccc}
\hline \hline & True \\
$m_{\mathrm{jj}}$ cut & Fraction & $p_{T}^{l_{1}}$ & $\Delta \phi_{\mathrm{jj}}$ & DNN \\
\hline$>850 \mathrm{GeV}$ & $6.66 \%$ & $6.67 \%_{-1.90 \%}^{+1.95 \%}$ & $6.67 \%_{-2.76 \%}^{+2.80 \%}$ & $6.66 \%_{-1.04 \%}^{+1.11 \%}$ \\
$>1200 \mathrm{GeV}$ & $6.68 \%$ & $6.70 \%_{-2.22 \%}^{+2.26 \%}$ & $6.70 \%_{-3.25 \%}^{+3.29 \%}$ & $6.68 \%_{-1.20 \%}^{+1.26 \%}$ \\
$>1500 \mathrm{GeV}$ & $6.67 \%$ & $6.71 \%_{-2.57 \%}^{+2.62 \%}$ & $6.68 \%_{-3.80 \%}^{+3.85 \%}$ & $6.67 \%_{-1.37 \%}^{+1.44 \%}$ \\
$>1800 \mathrm{GeV}$ & $6.69 \%$ & $6.70 \%_{-2.96 \%}^{+3.02 \%}$ & $6.68 \%_{-4.42 \%}^{+4.48 \%}$ & $6.69 \%_{-1.56 \%}^{+1.63 \%}$ \\
$>2000 \mathrm{GeV}$ & $6.66 \%$ & $6.67 \%_{-3.27 \%}^{+3.34 \%}$ & $6.66 \%_{-4.93 \%}^{+4.98 \%}$ & $6.66 \%_{-1.71 \%}^{+1.79 \%}$ \\
\hline \hline
\end{tabular}

particle-based DNN, dense DNN, BDT, $p_{T}^{l_{1}}$, and $\Delta \phi_{\mathrm{jj}}$, respectively. The DNN particle-based model has slightly more discriminative power comparing to BDT, and is much more powerful than the single variables, $p_{T}^{l_{1}}$ and $\Delta \phi_{\mathrm{jj}}$.

Fig. 3 shows several kinematic distributions of DNN inputs, and the distribution for the DNN discriminant itself. One can clearly see that the DNN greatly improves signalbackground discrimination compared to rectangular cuts.

We perform a fit to the DNN output and extract the LL fraction. The estimated LL fraction and accuracy is calculated by applying $2 \%$ luminosity uncertainty, and $5 \%$ systematic uncertainty both on LL and TT + TL. Table I shows the DNN results compared with methods based on $p_{T}^{l_{1}}$ and $\Delta \phi_{\mathrm{jj}}$. Examples of fit results can be seen in Fig. 4, for $m_{\mathrm{jj}}>1500 \mathrm{GeV}$, which are achieved by HistFactory [20] and cross-checked with RooFit [21].

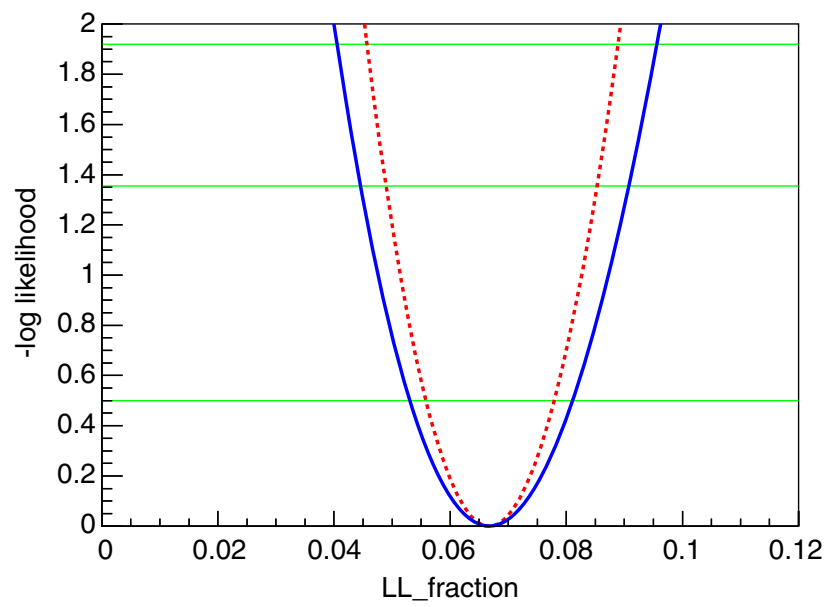

FIG. 4. Fit results for the LL fraction using particle-based DNN in $\mathrm{Mjj}>1500 \mathrm{GeV}$ region. The horizontal lines represent the $68 \%, 90 \%$, and $95 \%$ confidence level, from lower to upper. Apart from those, the solid and dotted lines are the log likelihood distributions, with or without systematics included. 


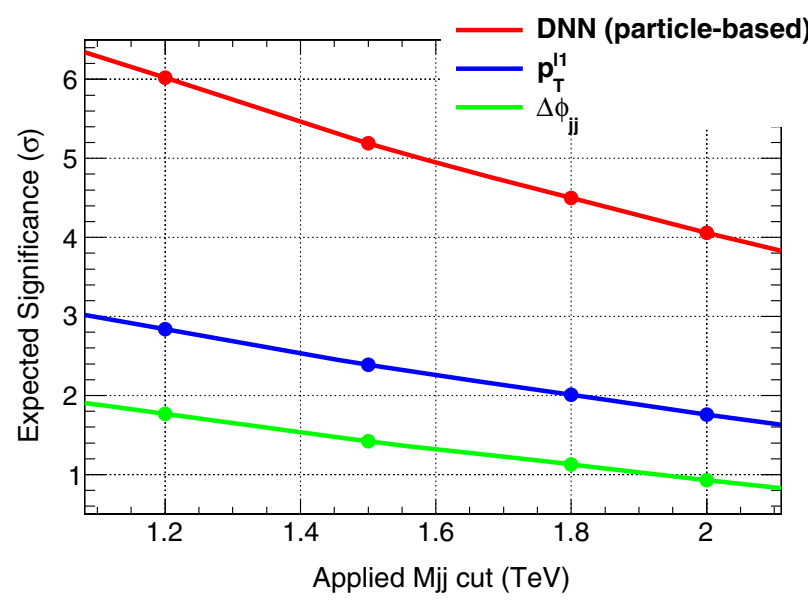

FIG. 5. Significance dependence on $m_{\mathrm{jj}}$ selection, through fitting $p_{T}^{l_{1}}, \Delta \phi_{\mathrm{jj}}$, or the DNN discriminant. Greatly improved performance is found with DNN.

Finally, we report here the significance. As mentioned above, the VBS $W^{ \pm} W^{ \pm}$process profits from lower background than in other VBS channels, considering that dominant backgrounds (WZ and hadron decays) are greatly suppressed and asymptotically negligible at high $m_{\mathrm{jj}}$ $[1,2,9]$. On the other hand, contributions from those dominant backgrounds can be estimated in experimental analysis and thus subtracted keeping uncertainties under control. In the ranges $m_{\mathrm{jj}}>1500$ and $2000 \mathrm{GeV}$, significances of 5.2 and 4.1 standard deviations can be achieved from a likelihood fit of DNN distributions. The same study has been performed via $p_{T}^{l_{1}}$ and $\Delta \phi_{\mathrm{jj}}$. Figure 5 shows greatly improved significance obtained with DNN.

In summary, measuring the longitudinally polarized fraction of $W^{ \pm} W^{ \pm}$scattering at the LHC is crucial to examine the unitarization mechanism of the vector boson scattering amplitude through Higgs and possible new physics. We apply here for the first time a deep neural network classification to extract the longitudinal fraction. Based on fast simulation implemented with the Delphes framework, significant improvement from DNN is found to be achievable and robust. An observation with an integrated luminosity of $3000 \mathrm{fb}^{-1}$ is found to reach 4 standard deviations at high $m_{\mathrm{jj}}$ region, such as above $2 \mathrm{TeV}$, where backgrounds are negligible. With a combination of the CMS and ATLAS measurements at the HL-LHC, an observation above 5 standard deviations can be expected with the deep learning technique proposed in this study.

\section{ACKNOWLEDGMENTS}

This work is supported in part by the National Natural Science Foundation of China, under Grants No. 11575005, by MOST under Grant No. 2018YFA0403900, and COST Action CA16108. We thank the CNRS/IN2P3 and the France China Particle Physics Laboratory (FCPPL) for their support. We also thank Gaël Touquet for his interesting suggestions about DNN, and Junjie Zhu and Pietro Govoni for their helpful discussions.
[1] A. M. Sirunyan et al. (CMS Collaboration), Phys. Rev. Lett. 120, 081801 (2018).

[2] The ATLAS Collaboration, Report No. ATLAS-CONF2018-030.

[3] M. J. G. Veltman, Acta Phys. Pol. B 8, 475 (1977); B. W. Lee, C. Quigg, and H. B. Thacker, Phys. Rev. Lett. 38, 883 (1977); B. W. Lee, C. Quigg, and H. B. Thacker, Phys. Rev. D 16, 1519 (1977).

[4] A. Alboteanu, W. Kilian, and J. Reuter, J. High Energy Phys. 11 (2008) 010.

[5] K. Doroba, J. Kalinowski, J. Kuczmarski, S. Pokorski, J. Rosiek, M. Szleper, and S. Tkaczyk, Phys. Rev. D 86, 036011 (2012).

[6] A. Freitas and J.S. Gainer, Phys. Rev. D 88, 017302 (2013).

[7] J. Searcy, L. Huang, M. A. Pleier, and J. Zhu, Phys. Rev. D 93, 094033 (2016).

[8] CMS Collaboration, Prospects for the study of vector boson scattering in same sign WW and WZ interactions at the HL-LHC with the upgraded CMS detector, CMS Physics Analysis Summary, CERN Report No. CMS-PAS-SMP-14-008, 2016, https://cds.cern.ch/ record/2220831.

[9] CMS Collaboration, Study of $W^{ \pm} W^{ \pm}$production via vector boson scattering at the HL-LHC with the upgraded CMS detector, CMS Physics Analysis Summary, CERN Report No. CMS-PAS-FTR-18-005, 2018, https://cds.cern.ch/ record/2646870.

[10] F. Chollet et al., https://github.com/fchollet/keras.

[11] Martín Abadi et al., TensorFlow: Large-scale machine learning on heterogeneous systems, 2015, Software available from tensorflow.org.

[12] P. Baldi, P. Sadowski, and D. Whiteson, Nat. Commun. 5, 4308 (2014).

[13] P. Baldi, P. Sadowski, and D. Whiteson, Phys. Rev. Lett. 114, 111801 (2015).

[14] B. P. Roe, H. J. Yang, J. Zhu, Y. Liu, I. Stancu, and G. McGregor, Nucl. Instrum. Methods Phys. Res., Sect. A 543, 577 (2005).

[15] A. Hocker et al., Proc. Sci., ACAT2007 (2007) 040. 
[16] J. Alwall, R. Frederix, S. Frixione, V. Hirschi, F. Maltoni, O. Mattelaer, H.-S. Shao, T. Stelzer, P. Torrielli, and M. Zaro, J. High Energy Phys. 07 (2014) 079.

[17] T. Sjostrand, L. Lonnblad, S. Mrenna, and P. Z. Skands, arXiv:hep-ph/0308153.

[18] J. de Favereau, C. Delaere, P. Demin, A. Giammanco, V. Lemaître, A. Mertens, and M. Selvaggi (DELPHES 3 Collaboration), J. High Energy Phys. 02 (2014) 057.

[19] D. L. Rainwater, R. Szalapski, and D. Zeppenfeld, Phys. Rev. D 54, 6680 (1996).
[20] K. Cranmer, G. Lewis, L. Moneta, A. Shibata, and W. Verkerke (ROOT Collaboration), Hist-Factory: A tool for creating statistical models for use with RooFit and RooStats, Report No. CERN-OPEN-2012-016, 2012, https://cds.cern .ch/record/1456844/.

[21] W. Verkerke and D. P. Kirkby, The RooFit toolkit for data modeling, in Proceedings of PHYSTAT 05: Statistical Problems in Particle Physics, Astrophysics and Cosmology, edited by L. Lyons and M. Karagoz (Oxford, England, 2005). 